\title{
The Design of Heat Exchangers
}

\author{
Arturo Reyes-León ${ }^{1}$, Miguel Toledo Velázquez ${ }^{1}$, Pedro Quinto-Diez ${ }^{1}$, Florencio Sánchez-Silva ${ }^{1}$, \\ Juan Abugaber-Francis ${ }^{1}$, Celerino Reséndiz-Rosas ${ }^{2}$ \\ ${ }^{1}$ Applied Thermal and Hydraulic Engineering Laboratory SEPI-ESIME-IPN Professional Unit, Lindavista, México D.F. \\ ${ }^{2}$ División de Estudios de Posgrado e Investigación, Instituto Tecnológico de Pachuca, \\ Pachuca de Soto Hidalgo, México \\ E-mail:mtv49@yahoo.com,arthuro_reyes@yahoo.com.mx \\ Received June 22, 2011; revised August 11, 2011; accepted August 25, 2011
}

\begin{abstract}
A relation between heat transferred and energy loss, for turbulent flow. In different tube arrangements, is made. The conditions are determined which decide the dimensions and velocities for a heat exchanger. Also, a reference to the economic dimensioning of heat exchangers is presented. In this study, the conditions which a heat exchanger must satisfy represent the best balance between the amounts of material employed. The investigation is restricted to the case of turbulent flow.
\end{abstract}

Keywords: Heat Changers, Energy Loss, Economic Dimensioning, Counterflow, Crossflow and Parallelflow

\section{Introduction}

The heat exchanger is an equipment that allows heat transference between two fluids at different temperatures. Heat exchangers are extensively used in industry due to their wide variety of construction and applications in heat transference processes for producing conventional energy such as condensers, heaters, boilers or steam generators. They provide an adequate surface for heat transference to occur and their mechanical and thermal characteristics allow high pressure and high temperature processes.

Heat exchangers are important, their optimization rises the competitiveness and allows energy saving. The necessity of saving and recovering energy for different processes in industry makes essential the develop of new manufacturing technology for heat exchangers in order to cover a wide range of operation conditions. In recent years, new software for design heat exchangers has been focus in adapting the equipment to the required process and new solutions have been found that make the design time shorter.

\section{Heat Transfer and Friction Work}

Let us first seek a relation between the heat transfer and energy loss. The problem is to determine the energy $E$ which has to be spent in order that a quantity of heat $Q$ may be transferred to a surface $F$, the average temperature difference being $\Theta[1,2]$.
Symbols used:

$Q=$ quantity of heat flowing per second.

$G=$ weight of gas or liquid flowing per second.

$\Theta=$ heat transfer coefficient.

$C p=$ specific heat at constant pressure.

$\Delta t=$ temperature variation of medium during flow.

$w=$ velocity of flow.

$\zeta=$ coefficient in pressure drop formula

$l=$ tube length.

$d=$ tube diameter.

$\gamma=$ specific weight.

$\mu=$ absolute viscosity.

$\lambda=$ thermal conductivity.

System units: $\mathrm{m}, \mathrm{kg}$, sec.

The heat given up to the surface $F$ is:

$$
Q=\alpha \cdot \Theta \cdot F
$$

And the gas loses a corresponding amount of heat

$$
Q=G \cdot C_{p} \cdot \Delta t
$$

whence

$$
\frac{\Delta t}{\Theta}=\frac{\alpha F}{G C_{p}}
$$

It is convenient to derive the expression of the energy loss first for longitudinal flow through the tubes, and applies it to the case of cross flow over a tube bank. The expression for the pressure drop in a tube is

$$
\Delta p=\zeta \frac{1}{d} \cdot \frac{w^{2}}{2 g} \gamma
$$


Putting the free gas section equal to $f$, and remembering that $G=w \gamma f$ and further that $\frac{1}{d}=\frac{F}{4 f}$ we obtain from Equation (3)

$$
\frac{\Delta p}{\Delta t}=\zeta \frac{w^{3} \gamma^{2} C p}{2 g \alpha}
$$

The ratio of the pressure drop in the heat transfer depends, therefore, of the velocity with which the heating surfaces are swept. If we group together those quantities which depend on the velocity, and introduce the Reynolds number $R e=\frac{w \gamma d}{\mu g}$ and the Nusselt number $N u=\frac{\alpha d}{\lambda}$ we obtain the following relation between heat transfer and pressure drop:

$$
\frac{\Delta p}{\Delta t / \Theta}=\frac{\mu^{3} g^{2} C p}{8 \gamma \lambda d^{2}} \cdot \zeta \cdot \frac{R e^{3}}{N u} .
$$

utting also:

$$
\zeta \frac{R e^{3}}{N u}=Z
$$

enables us to write the desired relation between the heat transfer and the energy loss in the case of longi tudinal flow through a tube, referred to $1 \mathrm{~kg}$ of medium flowing through the exchanger as

$$
\frac{\varepsilon}{\Delta t / \Theta}=\frac{\mu^{3} g^{2} C p}{8 \gamma \lambda d^{2}} \cdot z
$$

Similar expressions may be obtained for the case of a tube bank with cross flow, if $\zeta$ is taken as denoting the pressure drop coefficient per tube row, if the bank is $z_{1}$ the expression for the pressure drop becomes

$$
\Delta p=\zeta \cdot z_{1} \cdot \frac{w^{2}}{2 g} \gamma
$$

where $w$ is the velocity at the narrowest point between the tubes. If $s \cdot d$ denotes the pitch of the tubes across the flow, and $z_{q}$ denotes the number of tubes per row also in the direction across the flow, then with $F=\pi d l z_{q} \cdot z_{1}$ we obtain

$$
\frac{\Delta p}{\Delta t / \Theta}=\frac{w^{3} \gamma^{2} C p}{8 g \alpha} \cdot(s-1) \cdot \frac{4}{\pi} \zeta
$$

and introducing $\mathrm{Re}$ and $\mathrm{Nu}$ gives

$$
\frac{\Delta p}{\Delta t / \Theta}=\frac{w^{3} g^{2} C p}{8 \gamma \lambda d^{2}} \cdot(s-1) \cdot \frac{4}{\pi} \cdot \zeta \cdot \frac{R e^{3}}{N u}
$$

Let us put

$$
(s-1) \frac{4}{\pi} \cdot \zeta \cdot \frac{R e^{3}}{N u}=Z_{q}
$$

and we obtain a new form of Equation (7), for the case of cross flow:

$$
\frac{E}{\Delta t / \Theta}=\frac{\mu^{3} g^{3} C p}{8 \gamma^{2} \lambda d^{2}} \cdot Z_{q}
$$

giving the relation between the energy loss and the heat transfer for cross flow.

It is seen that the first term of the Equations (7) and (7a) contains characteristic quantities of the medium and the tube diameter. This means that for a given tube diameter the heat exchanger is fully characterized by the number $Z$. Now the pressure drop coefficient $\zeta$ is function of $R e$, while $N u$ is a function of $R e$ and of the Prandtl number, if we neglect the transition zone at the inlet, which is entirely permissible with cross flow exchangers many rows deep, or with longitudinal flow exchangers with relatively long tubes. But since the Prandtl number is purely a function of characteristic quantities of the medium, and we are interested only in a comparison of heat exchangers working with the same medium, and operating within the same temperature limits, $N u$ depends only on $R e$. This makes it possible to plot $N u$ as a function of $Z$. The $N u-Z$ diagram, therefore, gives a clear picture of the merit of a heat exchanger surface. Tube banks of different pitch are represented by different curves in the Nu-Z diagram. The higher a curve lies, the greater may be the heat transfer loading for a given energy expenditure, or, conversely, for a given surface and heat loading, the smaller the energy expenditure. Figure 1 which is drawn for gases contains curves relating to some of the most frequently used tube arrangements. In the case of cross flow, they are based on the values derived by Grimison from the tests of Pierson and Huge.

In order to make a comparison, curves for longitudinal flow have been inserted calculated with the aid of the formula given by Jung. It is seen that only at very high rates of heat transfer such as those which are achieved by the gas velocities attained in the Velox boiler, the longitudinal arrangement become more advantageous than the cross flow one. The curves giving the values of $Z$ which are plotted in Figure 1, in a logarithmic scale is almost straight. It is, therefore, permissible when considering segments of these curves, and without introducing any appreciable error to assume the following relation

$$
\log Z=B+m \log N u \text {. }
$$

or,

$$
Z=B N u^{m}
$$

where $B$ and $\mathrm{m}$ are constants whose value depends on the position in the diagram of the segment under consideration.

The expression for power loss can be written using 
Equation (7) and transforming Equation (3):

$$
L=\frac{\mu^{3} g^{2}}{8 \gamma^{2} d^{3}} \cdot F \cdot B \cdot N u^{m+1}
$$

1. Crossflow, staggered tubes, pitch $1.25 \times 1.25$.

2. Crossflow, tubes in line, pitch $1.25 \times 1.25$.

3. Crossflow, tubes in line, pitch $1.5 \times 1.5$.

4. Crossflow, tubes in line, pitch $2 \times 2$

5. Crosssflow, tubes in line, pitch $3 \times 3$.

6. Longitudinal flow in a tube.

7. Longitudinal flow between tubes, pitch $1.5 \times 1.5$.

8. Longitudinal tiny- between tubes, pitch $2 \times 2$.

9. Single tube in crossflow.

The pitch is expressed as times the tubes diameter.

The diagram shows for some common tube arrangements, the relation between the heat transfer number $N o$ and characteristic number $Z$ for the energy loss. For any given arrangement of the tubes, there is a definite value of $Z$ for every value of $N u$ of heat transfer, with the help of which the pressure drop and the exchanger surface may be obtained from the Equation (7) or (7a) [3].

\section{The Condition for the Right Exchanger}

The merit of an exchanger can only be judged when it is known what quantity of heat is equivalent to the mechanical energy which has to be supplied in the form of compressor or pump work to overcome the resistance of the exchanger. If the exchanger is an air preheater forming part of a steam power unit or a gas turbine then the overall efficiency of the plant or the efficiency when the exchanger is in service, determines the amount of the

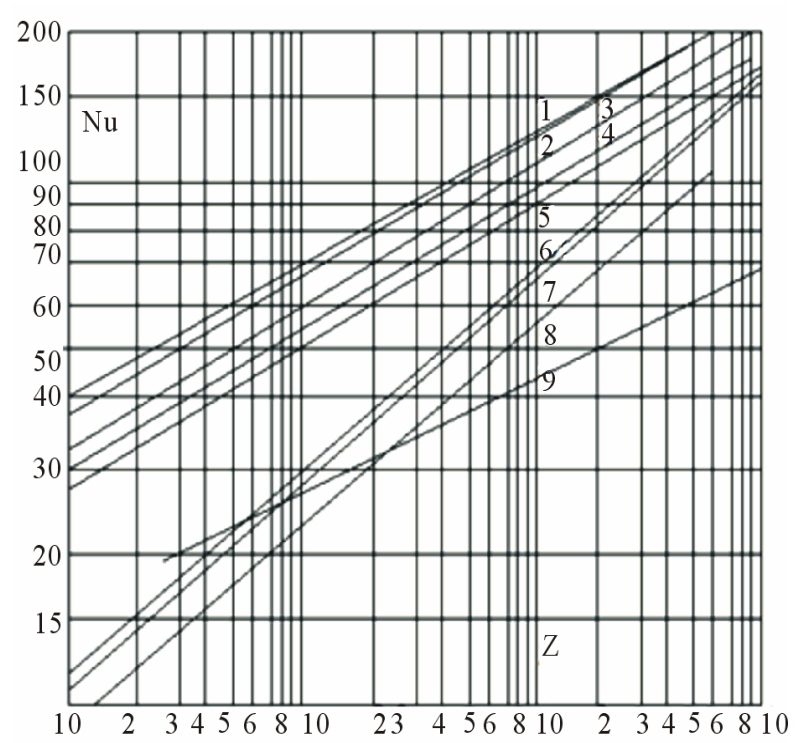

Figure 1. Relation between the energy loss and the heat transfer. energy (expressed in kcal), to the heat consumption required to produce this energy $[4,5]$.

On the other hand, if it is a case of plain heat transfer for instance, in blast heaters, furnaces, etc., where the energy absorbed in overcoming the resistance of the exchanger has to be supplied in the form of power purchased from an outside supply, then the cost of this power must be balanced against the cost of production of the more or less completely transferred heat in the exchanger. If $\eta$ denotes the efficiency of the plant with which the heat energy of the fuel is converted into mechanical energy, then the economic performance of the exchanger is given by

$$
\text { Useful }=Q-\frac{A L}{\eta} .
$$

useful heat is, therefore, equal to the difference of the heat transferred and the heat required for the production of the mechanical work absorbed.

The right heat exchanger is, therefore, the exchanger which with a given surface and with a given diameter of tubes results in a maximum amount of useful heat. The condition for this is

$$
\eta d Q-A d L=0
$$

We shall now seek expressions for $d Q$ and $d L$ in terms of $N u$.

Let the suffix 1 denote the hot medium, and the suffix 2 the cold one. The meaning of the symbols is made clear by the Figure 2. We may write for the temperature variation of the hot medium

$$
t_{1}^{\prime}-t_{1}^{\prime \prime}=\varepsilon_{1} \cdot\left(t_{1}^{\prime}-t_{2}^{\prime}\right) .
$$

and similarly, that of the colder one is

$$
t_{2}^{\prime}-t_{2}^{\prime \prime}=\varepsilon_{2} \cdot\left(t_{1}^{\prime}-t_{2}^{\prime}\right) .
$$

Further, let the mean temperature difference be given by

$$
\Theta=a \cdot\left(t_{1}^{\prime}-t_{2}^{\prime}\right) .
$$

The factor a depends only on $\varepsilon_{1}$ and $\varepsilon_{2}$, and is plotted in Figure 3 for counter flow, for cross flow and parallel flow, for the case $\varepsilon_{1}=\varepsilon_{2}$. If we denote by $k$ the overall heat transfer coefficient, the heat transferred is given by

$$
Q=a \cdot k \cdot F\left(t_{1}^{\prime}-t_{2}^{\prime}\right)
$$

For a small change in the rate of heat transfer we have.

$$
d Q=\left(a+k F \frac{d a}{d k F}\right) \cdot d k F \cdot\left(t_{1}^{\prime}-t_{2}^{\prime}\right) .
$$

Since $k F$ may be treated as a single quantity.

We put

$$
d Q=b \cdot d k F \cdot\left(t_{1}^{\prime}-t_{2}^{\prime}\right)
$$




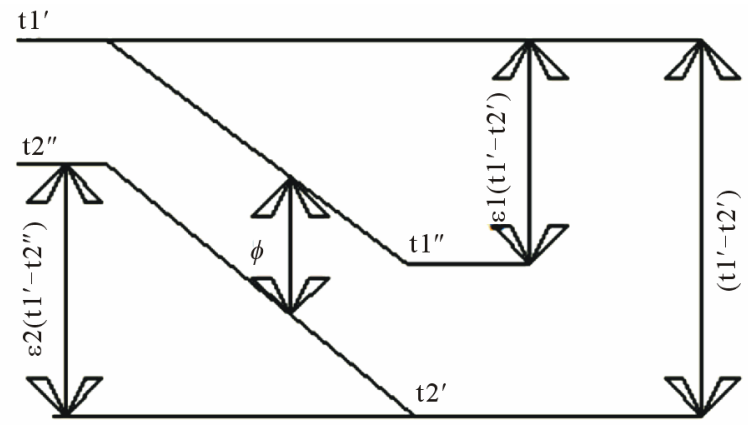

Figure 2. Diagram of temperature variation in an exchanger.

where $b$ depends only on $\varepsilon_{1}$ and $\varepsilon_{2}$. From Equations (11) and (12), and from the relation $Q=\varepsilon G C p\left(t_{1}^{\prime}-t_{2}^{\prime}\right)$, it is found that

$$
b=\frac{a}{1-\frac{\varepsilon}{a} \frac{d a}{d \varepsilon}}
$$

Curves for $b$ are given in Figure 3. In the case of heat transfer in metal exchangers the thermal resistance of the exchanger wall may be neglected without introducing any appreciable error. Hence, it is permissible to write

$$
\frac{1}{k F}=\frac{1}{\alpha_{1} F_{1}}+\frac{1}{\alpha_{2} F_{2}}
$$

Differentiating and introducing the Nusselt number in place of the quantities $d \alpha_{1}$ and $d \alpha_{2}$ gives

$$
d k F=\left(\frac{k F}{\alpha_{1} F_{1}}\right)^{2} \cdot F_{1} \cdot \frac{\lambda_{1}}{d_{1}} \cdot d N u_{1}+\left(\frac{k F}{\alpha_{2} F_{2}}\right)^{2} \cdot F_{2} \cdot \frac{\lambda_{2}}{d_{2}} \cdot d N u_{2}
$$

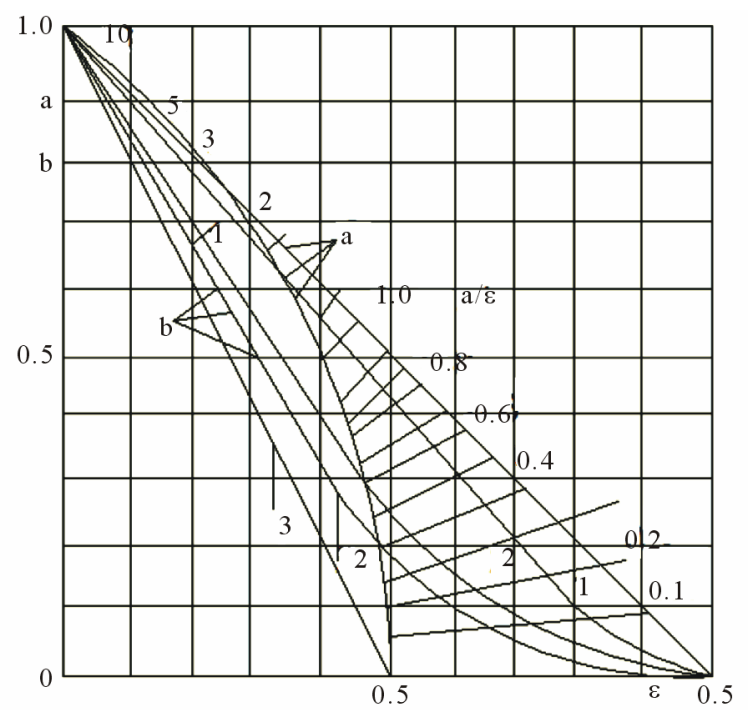

Figure 3. Characteristic numbers $a$ and $b$ of Equations (11) and (12) for $\varepsilon_{1}=\varepsilon_{2}$ in the case of counterflow, crossflow and parallelflow.
The total power loss is the sum of the losses for the hot and cold mediums. We use Equation (9) and put

$$
F \frac{\mu^{3} g^{2} B}{8 \gamma^{2} d^{3}}=P
$$

and obtain for the total energy loss

$$
L=P_{1} N u_{1}^{m_{1}+1}+P_{2} N u_{2}^{m_{2}+1} .
$$

The change in loss with change of velocity is then given by

$$
d L=P_{1}\left(m_{1}+1\right)_{1} N u_{1}^{m_{1}} d N u_{1}+P_{2}\left(m_{2}+1\right) N u_{2}^{m_{2}} d N u_{2}
$$

The change in heat quantity transferred with change of specific loading $k F$ is given by Equation (12). Inserting Equations (12) and (17) in Equation (10) and taking into account Equation (15) gives

$$
\begin{aligned}
& \frac{\eta b}{A}\left(t_{1}^{\prime}-t_{2}^{\prime}\right)\left[\left(\frac{k F}{\alpha_{1} F_{1}}\right)^{2} \cdot F_{1} \cdot \frac{\lambda_{1}}{d_{1}} \cdot d N u_{1}\right. \\
& \left.+\left(\frac{k F}{\alpha_{2} F_{2}}\right)^{2} \cdot F_{2} \cdot \frac{\lambda_{2}}{d_{2}} \cdot d N u_{3}\right] \\
& -P_{1}\left(m_{1}+1\right) N u_{1}^{m_{1}} d N u_{1}-P_{2}\left(m_{2}+1\right) N u_{2}^{m_{2}} d N u_{2}=0
\end{aligned}
$$

The coefficients of $d N u_{1}$ and $d N u_{2}$ must be equal 0 . This gives two new equations namely,

$$
\begin{aligned}
& \frac{\eta b}{A}\left(t_{1}^{\prime}-t_{2}^{\prime}\right)\left[\left(\frac{k F}{\alpha_{1} F_{1}}\right)^{2} \cdot F_{1} \cdot \frac{\lambda_{1}}{d_{1}}\right]=P_{1}\left(m_{1}+1\right) N u_{1}^{m_{1}} \\
& \frac{\eta b}{A}\left(t_{1}^{\prime}-t_{2}^{\prime}\right)\left[\left(\frac{k F}{\alpha_{2} F_{2}}\right)^{2} \cdot F_{2} \cdot \frac{\lambda_{2}}{d_{2}}\right]=P_{2}\left(m_{2}+1\right) N u_{2}^{m_{2}}
\end{aligned}
$$

Dividing Equation 19 (b) by Equation 19(a), inserting for $P_{1}$ and $P_{2}$ the values given by Equation (16) and substituting $\mathrm{Nu}$ for $\alpha_{1}$ and $\alpha_{2}$ gives

$$
\frac{N u_{2}^{m_{2}+2}}{N u_{1}^{m_{1}+2}}=\frac{\left(m_{1}+1\right) \cdot B_{1} \cdot F_{1}^{2} \mu_{1}^{3} \gamma_{2}^{2} \lambda_{1} d_{2}^{4}}{\left(m_{2}+1\right) \cdot B_{2} \cdot F_{2}^{2} \mu_{2}^{3} \gamma_{1}^{2} \lambda_{2} d_{1}^{4}}
$$

This equation determines the ratio of the velocities in the right heat exchanger; it does not, however, say anything about the absolute value of the velocities. It means that heat exchangers in which this ratio of the velocities is observed have for a given surface and a given heat quantity the lowest friction loss.

If we take the roots of Equations 19(a) and 19(b), and remembering that $\frac{k F}{\alpha_{1} F_{1}}+\frac{k F}{\alpha_{2} F_{2}}=1$ we obtain as the second condition for the right heat exchanger:

$$
\sqrt{\frac{\eta}{A} b\left(t_{1}^{\prime}-t_{2}^{\prime}\right)}=\sqrt{R_{1} N u_{1}^{m_{1}}}+\sqrt{R_{2} N u_{2}^{m_{2}}}
$$


The factor $R$ contains only constants.

$$
R=(m+1) \frac{\mu^{3} g^{2} B}{8 \gamma^{2} d^{2} \lambda} .
$$

Two Equations (20) and (21) completely determine the most favourable heat exchanger. The resulting transcenddental equation must be solved by trial. The surfaces are found with the aid of the numbers $N u$, and $N u$, and since $N u$ is a function of the Reynolds number, the velocities $\mathrm{w}_{1}$ and $\mathrm{w}_{2}$ are also determinate. The dimensions of the heat exchanger are, therefore, fixed.

\section{The Economic Dimensioning of a Heat Exchanger}

It was seen in the first part that there is a function $Z$ which serves as a criterion of the merit of tube arrangements in heat exchangers. In the second part the conditions fix the dimensions of the surface and sections of the right heat exchanger. The exchanger should, however, like every other apparatus be correctly dimensioned from the economic point of view, that is the total sum of the capital charges and of the running costs should be a minimum.

If $P$ denotes the capital cost, $\mathrm{n}$ the interest and deprecition rate, then the capital chargers are

$$
K_{1}=n P \text {. }
$$

and if $L$ is the power absorbed, $\eta$ the efficiency, $h$ the operating hours in one year and $p$ the price per kilowatt-hour, then the power costs are

$$
K_{2}=\operatorname{Lh} \frac{1}{\eta} p .
$$

and the total costs in one operational year

$$
K_{1}+K_{2}=n P+h \frac{1}{\eta} p L .
$$

there should be a minimum hence,

$$
n P+h \frac{1}{\eta} p L=0
$$

The capital costs will increase approximately in proportion to the exchanger surface, and for a given tube diameter, inversely proportionally to the heat transfer number, or

$$
F=N u^{-1}, P=N u^{-1} .
$$

But according to Equation (9), the energy proportional to $F N u^{m+1}$; hence substituting for $F=N u^{-1}$

$$
L=C N u^{m} \text { or } P \sim L^{-\frac{1}{m}} .
$$

differentiating and dividing by $P$

$$
\frac{d P}{P}+\frac{1}{m} \frac{d L}{L}=0
$$

Dividing Equation (22) by Equation (23)

$$
n P+h \frac{1}{\eta} p m L=0
$$

The total costs in an operational year are a minimum when the capital charges amount to m-times the power costs. Within the range of practical application, that is, for $N u=40$ to 120 the curve which is over this range can be seen upon as a straight line, for instance curve 3 (cross flow heat exchanger with a tube pitch $1.5 \times 1.5$ ) gives an exponent $m=3.84$ and a constant $B=166$; for curve 7 (longitudinal flow with a tube pitch $1.5 \mathrm{~d}$ ) the figures are $m=2.67$ and $B=99500$.

The starting point for this study has been the as sumption of a fixed tube diameter and tube pitch. These and the choice between staggered or straight arrangement of the tubes are determined by dirt deposit and cleaning considerations. How closely these assumptions and the results of the calculation of the right heat exchanger may be adhered to in practice depends on manufacturing conditions, but in any case the above exposition serves as a guide to show in what direction and to what extent modifications are desirable.

\section{Example}

Designing a compact heat exchanger for heating wings continuous $20,000 \mathrm{~kg} / \mathrm{h}$ of air from an inlet temperature ( $\left.\mathrm{T}_{\text {iair }}\right) 10^{\circ} \mathrm{C}$ to an out temperature (air $\mathrm{T}_{\mathrm{o}}$ air) of $55^{\circ} \mathrm{C}$ heating fluid used water volumetric flow $\dot{V}_{w}\left(10 \mathrm{~m}^{3} / \mathrm{h}\right.$ at temperature $\mathrm{T}$ of $95^{\circ} \mathrm{C}$, the input date are shown in Table $1[6,7]$.

The density and heat capacity of both fluids are obtained from tables to standard atmosphere conditions. The characteristic of tube and of the wins are shown in Table 2.

Diagram inlets from primary and secondary of the compact heat exchanger are show in Figure 4.

\section{Unit thermal water consumption}

$$
\begin{gathered}
q_{\mathrm{t} \text { agua }}=\dot{m}_{\text {agua }} c p_{\text {agua }}=\dot{V}_{\text {agua }} \rho_{\text {agua }} c p_{\text {agua }} \\
q_{t \text { agua }}=10 \frac{\mathrm{m}^{3}}{\mathrm{~h}} \times \frac{1 \mathrm{~h}}{3600 \mathrm{~s}} \times 962.036 \frac{\mathrm{kg}}{\mathrm{m}^{3}} \times 4217 \frac{\mathrm{J}}{\mathrm{kgC}} \\
=11269,18 \frac{\mathrm{W}}{0_{C}}=11.269 \frac{\mathrm{kW}}{0_{C}}
\end{gathered}
$$

Unit thermal air consumption

$$
q_{\mathrm{t} \text { aire }}=\dot{m}_{\text {aire }} c p_{\text {aire }}
$$


Table 1. Input data of the compact heat exchanger.

\begin{tabular}{lcc}
\hline \multicolumn{3}{c}{ Input data } \\
\hline $\mathrm{T}_{\text {inlet }}$ of Secondary fluid (Tfi) & 10.00 & ${ }^{\circ} \mathrm{C}$ \\
$\mathrm{T}_{\text {out }}$ of Secondary fluid (Tfo) & 55.00 & ${ }^{\circ} \mathrm{C}$ \\
$\mathrm{T}_{\text {inlet }}$ of Primary fluid (Tci) & 95.00 & ${ }^{\circ} \mathrm{C}$ \\
velocity primary fluid (Wp) & 1.00 & $\mathrm{~m} / \mathrm{s}$ \\
velocity secondary fluid (Ws) & 3.00 & $\mathrm{~m} / \mathrm{s}$ \\
Mass flow of primary fluid (mp) & 2.77 & $\mathrm{~kg} / \mathrm{s}$ \\
Mass flow of secondary fluid (ms) & 5.55 & $\mathrm{~kg} / \mathrm{s}$ \\
heat capacity primary fluid (Cpp) & 4217 & $\mathrm{~J} / \mathrm{kg}-{ }^{\circ} \mathrm{C}$ \\
heat capacity secondary fluid (Cps) & 1005.00 & $\mathrm{~J} / \mathrm{kg}-{ }^{\circ} \mathrm{C}$ \\
density primary fluid ( $\rho$ p) & 962.04 & $\mathrm{~kg} / \mathrm{m}^{3}$ \\
density secondary fluid $(\rho \mathrm{s})$ & 1.15 & $\mathrm{~kg} / \mathrm{m}^{3}$ \\
\hline
\end{tabular}

Table 2. Characteristic of tube and of the wing.

\begin{tabular}{|c|c|c|}
\hline \multicolumn{3}{|c|}{ Characteristic of tube } \\
\hline Outside diameter of tube (do) & 0.012700 & $\mathrm{~m}$ \\
\hline Inside diameter of tube (di) & 0.011280 & $\mathrm{~m}$ \\
\hline $\begin{array}{l}\text { Wheelbase of the tubes in the direction } \\
\text { of the tubes (1) }\end{array}$ & 0.080 & $\mathrm{~m}$ \\
\hline $\begin{array}{l}\text { Wheelbase of the tubes in the direction } \\
\text { of height (h) }\end{array}$ & 0.040 & $\mathrm{~m}$ \\
\hline Area inside of the tube (At-int) & $9.993 \mathrm{E}-505$ & $\mathrm{~m}^{2}$ \\
\hline \multicolumn{3}{|c|}{ Characteristics of the wing } \\
\hline Thickness of the wing (e) & $3.00 \mathrm{E}-04$ & $\mathrm{~m}$ \\
\hline Thermal conductivity (Aluminum, $\mathrm{k}$ ) & 203.52 & $\mathrm{~W} / \mathrm{m}^{\circ} \mathrm{C}$ \\
\hline
\end{tabular}

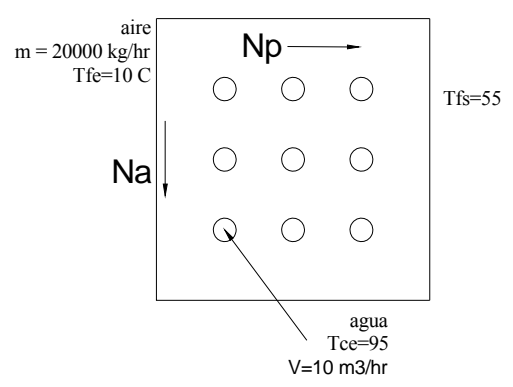

Figure 4. Diagram inlets from primary and secondary fluids.

$$
\begin{aligned}
q_{t \text { aire }} & =20000 \frac{\mathrm{kg}}{\mathrm{h}} \times \frac{1 \mathrm{~h}}{3600 \mathrm{~s}} \times 1.005 \frac{\mathrm{kJ}}{\mathrm{kgK}} \\
& =5.577 \frac{\mathrm{kW}}{\mathrm{C}} \\
& \mathrm{T}_{m \text { aire }}=\frac{55+10}{2}=32.5^{\circ} \mathrm{C}
\end{aligned}
$$

The density of air to Tm is show in Table 3.
Table 3. Density of air to Tm.

\begin{tabular}{ll}
\hline $\mathrm{T}\left({ }^{\circ} \mathrm{C}\right)$ & $\rho\left(\frac{\mathrm{kg}}{\mathrm{m}^{3}}\right)$ \\
30 & 1.165 \\
32.5 & $\rho_{\text {air }}=1.15\left(\frac{\mathrm{kg}}{\mathrm{m}^{3}}\right)$ \\
40 & 1.128 \\
\hline
\end{tabular}

\section{Themal flow}

$$
\begin{aligned}
& \dot{Q}=q_{\mathrm{t} \text { air }}\left(\mathrm{T}_{\mathrm{s} \text { air }}-\mathrm{T}_{\text {e air }}\right)=\dot{m}_{\text {air }} c p_{\text {air }}\left(\mathrm{T}_{\mathrm{s} \text { air }}-\mathrm{T}_{\mathrm{e} \text { air }}\right) \\
& \dot{Q}=5577,75 \frac{\mathrm{W}}{{ }^{\circ} \mathrm{C}} \\
& \left(55^{\circ} \mathrm{C}-10^{\circ} \mathrm{C}\right)=250.99 \mathrm{KW}=250998,75 \mathrm{~W}
\end{aligned}
$$

Temperature of exit of the water

$$
\begin{aligned}
\mathrm{T}_{\text {s water }} & =\mathrm{T}_{\text {e water }}-\frac{\dot{Q}}{q_{\mathrm{twater}}} \\
& =95^{\circ} \mathrm{C}-\frac{250998,75 \mathrm{~W}}{11269,18 \mathrm{~W} /{ }^{\circ} \mathrm{C}}=73.51^{\circ} \mathrm{C}
\end{aligned}
$$

Velocities are: $2.5 \mathrm{~m} / \mathrm{s}$ and $3.5 \mathrm{~m} / \mathrm{s}$

$$
\begin{gathered}
W_{\text {water }}=1 \mathrm{~m} / \mathrm{s} \\
W_{\text {air }}=3 \mathrm{~m} / \mathrm{s}
\end{gathered}
$$

\section{Logarithmic mean temperature}

$$
\begin{aligned}
\Delta T_{m l}=\frac{\Delta \theta_{1}-\Delta \theta_{2}}{\ln \frac{\Delta \theta_{1}}{\Delta \theta_{2}}} & =\frac{(95-55)-(73.51-10)}{\ln \frac{40}{63.51}} \\
\Delta T_{m l} & =50.85^{\circ} \mathrm{C}
\end{aligned}
$$

\section{Surface airflow}

$$
\begin{gathered}
S_{p}=\frac{\dot{V}_{\text {air }}}{W_{\text {air }}} \\
\dot{V}_{\text {air }}=\frac{W_{\text {air }}}{\rho_{\text {air }}}
\end{gathered}
$$

Table gives the density of dry air at a temperature of $58.38^{\circ} \mathrm{C}$

$$
\rho_{\text {air }}=1.15 \frac{\mathrm{kg}}{\mathrm{m}^{3}}
$$

Volumetric air flow

$$
\dot{V}_{\text {air }}=\frac{20000 \frac{\mathrm{kg}}{\mathrm{h}} \times \frac{1 \mathrm{~h}}{3600 \mathrm{~s}}}{1.15 \frac{\mathrm{kg}}{\mathrm{m}^{3}}}=4.83 \frac{\mathrm{m}^{3}}{\mathrm{~s}}
$$




$$
\begin{gathered}
S_{p}=\frac{4.83 \frac{\mathrm{m}^{3}}{\mathrm{~s}}}{3 \frac{\mathrm{m}}{\mathrm{s}}}=1.61 \mathrm{~m}^{2} \\
\frac{d_{\text {int }}}{d_{\text {out }}}=\frac{11.66 \mathrm{~mm}}{15.88 \mathrm{~mm}} \quad h=40 \mathrm{~mm}
\end{gathered}
$$

\section{Surface water flow}

$$
\begin{gathered}
S_{T}=\frac{\pi}{4} d_{\text {int }}{ }^{2} N_{A} ; \quad W_{\text {water }}=1 \mathrm{~m} / \mathrm{s} \\
S_{T}=\frac{\dot{V}_{\text {water }}}{\mathrm{W}_{\text {water }}}=\frac{10 \frac{\mathrm{m}^{3}}{h} \times \frac{1 h}{3600 \mathrm{~s}}}{1 \mathrm{~m} / \mathrm{s}}=2.88 \times 10^{-3} \mathrm{~m}^{2}
\end{gathered}
$$

Number of tubes in the direction of height $(H)$ heat exchanger

$$
\begin{aligned}
N_{A} & =\frac{S_{T}}{A_{\text {tube,inlet. }}}=\frac{4}{\pi} \frac{S_{T}}{d_{\text {inlet }}^{2}} \\
& =\frac{4 \times 2.78 \times 10^{-3} \mathrm{~m}^{2}}{\pi(0.01128) \mathrm{m}^{2}}=29 \text { tubes }
\end{aligned}
$$

\section{Height of heat exchanger}

$$
H=h \cdot N_{A}=0.040 \mathrm{~m} \cdot 29=1.15 \mathrm{~m}
$$

Winged tube length

$$
S_{p}=H \cdot L a \therefore L a=\frac{S_{p}}{H}=\frac{1.61 \mathrm{~m}^{2}}{1.15 \mathrm{~m}}=1.40 \mathrm{~m}
$$

In Table 4 are shown calculated values of $\mathrm{Na}, \mathrm{H}$ and $\mathrm{L}$ to the compact heat exchanger.

Wing thickness, this value is assumed by the types of materials available in the industry

$$
e=0.3 \mathrm{~mm}
$$

Calculation of Reynolds number (Re), Nusselt (Nu) and the convective coefficient (hi) of primary fluid

Whereas, $d h=d i$; and the Prandtl Number (Prp), dynamic viscosity ( $\mu p)$ and conductivity of the primary fluid are determined from tables to the average temperature of primary fluid (Tmp). These values are shown in Table 5.

$$
\begin{gathered}
\operatorname{Rep}=\frac{W p \cdot d h}{\mu p} \\
N u=0.023 \cdot \operatorname{Rep}^{0.8} \cdot \operatorname{Prp}^{0.3} \\
h i=\frac{N u \cdot k p}{d h}
\end{gathered}
$$

The values of Reynolds number, Nusselt number and convection coefficient inside of primary fluid are shown in Table 6.
Calculation of the mass velocity and maximum mass velocity of the secondary fluid.

$$
\begin{gathered}
s=\frac{h}{d e} \\
W m s=W s \cdot \rho s \\
W m s^{\prime}=W m s\left(\frac{s}{s-1}\right)
\end{gathered}
$$

The values of mass velocity, maximum mass velocity and relationship s are shown in Table 7

Calculation of hydraulic diameter $\left(d h^{\prime}\right)$, Reynolds $(R e)$ and convection coefficient $(I)$ as a function the secondary fluid pitch between wing $(p)$ Tables obtained forthe Prandtl number $(P r)$, the dynamic viscosity $(\mu s)$ and thermal conductivity $(\mathrm{ks})$ of secondary fluid at film temperature (Tmp). These values are shown in Table 8.

Table 4. Values of $N a, H$ and $L$ to the compact heat exchanger.

\begin{tabular}{lcc}
\hline Number of tubes in direction of height $(\mathrm{Na})$ & 29 & Tubos \\
\hline Exchanger height $(H)$ & 1.15 & $\mathrm{~m}$ \\
Overall longitude of the tube $(L)$ & 1.40 & $\mathrm{~m}$ \\
\hline
\end{tabular}

Table 5. Values of Prp, $v$ and $k p$.

\begin{tabular}{lll}
\hline Prandtl number of primary fluid (Prp) & 2.11 & \\
Viscosity of primary fluid $(v)$ & $3.50 \mathrm{E}-07$ & $\mathrm{~m}^{2} / \mathrm{s}$ \\
Conductivity of primary fluid $(k p)$ & 0.68 & $\mathrm{w} / \mathrm{m}{ }^{\circ} \mathrm{C}$ \\
\hline
\end{tabular}

Table 6. Values of Rep, Nup and hi to the compact heat exchanger.

\begin{tabular}{lll}
\hline Reynolds number of primary fluid (Rep) & 32228.57 & \\
Nusselt number of fluid primary (Nup) & 116.31 & \\
Convection coefficient inside (hi) & 6972.23 & $\mathrm{~W} / \mathrm{m}^{2}-\mathrm{K}$ \\
\hline
\end{tabular}

Table 7. Values of $s, W m s$ and $W m s$ of the secondary fluid.

\begin{tabular}{llll}
\hline Relationship between $\mathrm{h}$ and the outer diameter $(s)$ & 2.36 & \\
Mass velocity of secondary fluid $(W m s)$ & 3.45 & $\mathrm{~kg} / \mathrm{s}^{-\mathrm{m}^{2}}$ \\
Maximus velocity of secondary fluid $\left(W m s^{\prime}\right)$ & 5.98 & $\mathrm{~kg} / \mathrm{s}^{2} \mathrm{~m}^{2}$ \\
\hline
\end{tabular}

Table 8. Values of $T p, \operatorname{Pr}, \rho s$ and $k s$ of secondary fluid.

\begin{tabular}{lll}
\hline Film Temperature (Tp) & 58.33 & ${ }^{\circ} \mathrm{C}$ \\
Prantl Number of secondary fluid (Prs) & 0.6963 & \\
Dynamic Viscosity of secondary fluid $(\rho s)$ & 0.0781 & $\mathrm{~kg} / \mathrm{m}-\mathrm{s}$ \\
Conductividad del flujo secundario $(k s)$ & 0.0287 & $\mathrm{w} / \mathrm{m}{ }^{\circ} \mathrm{C}$ \\
\hline
\end{tabular}




$$
\begin{gathered}
d h^{\prime}=2 p\left(1-\frac{\pi \cdot d e^{2}}{4 h \cdot l}-\frac{e}{p}\right) \\
\operatorname{Re}=\frac{W m s^{\prime} \cdot d h^{\prime}}{\mu s} \\
N u=0.26 \cdot R e^{0.6} \cdot \operatorname{Prp}^{1 / 3} \\
h e=\frac{N u \cdot k s}{d h^{\prime}}
\end{gathered}
$$

Table 9. show the values of $m, d h^{\prime}, R e, N u$ and he of the secondary fluid.

Calculation of equivalent diameter (dea) and efficiencies of wings. (Table 10)

In Table 11 show characteristics of wing, Aluminum considering.

$$
\begin{gathered}
d e a=\frac{2 \cdot h \cdot l}{h+l} \\
\mu=\frac{d e a}{d e} \\
\beta=\sqrt{\frac{2 \cdot h e}{k s \cdot e}}
\end{gathered}
$$

$\left[d e \cdot \beta \cdot \frac{\mu-1}{2}\right]$, Relationship for the efficiencies.

Calculation of the ratio number of wings with borders with the total number of wings.

Considering that $N=N a \times N p$ where $\mathrm{Na}$ is the number of tubes in direction of height and $N p$ is the number of tubes in the direction of secondary flow.

Number of wings without borders

$$
* N 0=N a \cdot N p-2(N a+N p)+4
$$

Number of wings with a border

$$
\text { * } N 1=2(N a+N p)-8
$$

Number of wings with two borders

\begin{tabular}{|c|c|c|c|c|}
\hline Pitch $(m)$ & Hydraulic Diameter $\left(d h^{\prime}\right)$ & Reynolds Number secondary $(R e)$ & Nusselt Number secondary $(\mathrm{Nu})$ & Convective Coefficient secondary (he) \\
\hline 0.002 & $3.08 \mathrm{E}-03$ & 718.4305 & 11.923624 & 110.9873 \\
\hline 0.003 & $4.92 \mathrm{E}-03$ & 1147.548 & 15.792099 & 92.027767 \\
\hline 0.004 & $6.77 \mathrm{E}-03$ & 1576.665 & 19.108255 & 81.04597 \\
\hline 0.005 & $8.61 \mathrm{E}-03$ & 2005.782 & 22.077386 & 73.606079 \\
\hline 0.006 & $1.04 \mathrm{E}-02$ & 2434.899 & 24.800817 & 68.113749 \\
\hline 0.007 & $1.23 \mathrm{E}-02$ & 2864.017 & 27.337746 & 63.831783 \\
\hline 0.008 & $1.41 \mathrm{E}-02$ & 3293.134 & 29.726447 & 60.364755 \\
\hline
\end{tabular}

$$
* N 2=4
$$

\begin{tabular}{|c|c|c|c|c|}
\hline Pitch $(m)$ & $\begin{array}{c}\text { Convective Coefficient } \\
\text { secondary }(h e)\end{array}$ & $\begin{array}{c}\text { Equivalence Factor } \\
\text { wings }(\beta)\end{array}$ & $d e a^{* *}=\left(d e^{*} \beta\right) *(\mu-1 / 2)$ & Efficiency obtained from the graphic $\eta f$ \\
\hline 0.002 & 110.9873 & 60.295115 & 0.823028315 & 0.9 \\
\hline 0.003 & 92.027767 & 54.904119 & 0.749441229 & 0.92 \\
\hline 0.004 & 81.04597 & 51.524193 & 0.703305238 & 0.93 \\
\hline 0.005 & 73.606079 & 49.102356 & 0.670247159 & 0.95 \\
\hline 0.006 & 68.113749 & 47.234887 & 0.644756207 & 0.96 \\
\hline 0.007 & 63.831783 & 45.726081 & 0.624161001 & 0.97 \\
\hline 0.008 & 60.364755 & 44.466936 & 0.606973681 & 0.98 \\
\hline
\end{tabular}

*Note: This means that the equations are modified as a function on $N p$. (Table 12)

$$
\psi 0=\frac{N 0}{N} \quad \psi 1=\frac{N 1}{N} \quad \psi 2=\frac{N 2}{N}
$$

Calculation of correction coefficients for the wings. (Table 13)

$$
\begin{gathered}
C_{0}=1 \\
C_{1}=1+0.5\left[\frac{h_{e}}{k_{a l}}\left(\frac{l}{h}+\frac{h}{l}\right)\right]^{1 / 4} \\
C_{2}=1+\left[\frac{h_{e}}{k_{a l}}\left(\frac{l+h}{h}\right)\right]^{1 / 3}
\end{gathered}
$$

Table 9. Values of $m, d h^{\prime}, R e, N u$ and $h e$ of the secondary fluid.

Table 10. Values of equivalent diameter and efficiencies of wings. 
Table 11. Characteristics of Aluminum.

\begin{tabular}{lcc}
\hline Thermal conductivity of aluminum $(k)$ & 203.525 & $\mathrm{w} / \mathrm{mC}$ \\
Wing thickness $(e)$ & $3.00 \mathrm{E}-04$ & $\mathrm{~m}$ \\
\hline
\end{tabular}

Table 12. Relations with number of wings on the total number of wings.

\begin{tabular}{|c|c|c|}
\hline \multicolumn{3}{|c|}{ Relations with number of wings on the total number of wings } \\
\hline$\psi$ & $(N p)=1$ & $(\mathrm{~Np})=2 \quad(\mathrm{~Np})=3 \quad(\mathrm{~Np})=4 \quad(\mathrm{~Np})=5 \quad(\mathrm{~Np})=6$ \\
\hline$\tilde{\psi_{0}}$ & 0 & 0.31019520 .46529280 .55835140 .620390394 \\
\hline$\psi_{1}$ & 0 & 0.41388290 .35647147 \\
\hline \multicolumn{3}{|c|}{$\psi_{2} 0.9305855910 .0694140 .04627630 .03470720 .02776580 .023138136$} \\
\hline
\end{tabular}

Table 13. Correction factors for each type of wing.

\begin{tabular}{ccccc}
\hline \multicolumn{5}{c}{ Correction factors for each type of wing } \\
\hline Pitch $(m)$ & $h_{e}$ & $c_{0}$ & $c_{1}$ & $c_{2}$ \\
0.002 & 110.987 & 1 & 1.510965 & 2.029347 \\
0.003 & 92.0278 & 1 & 1.487588 & 1.967038 \\
0.004 & 81.046 & 1 & 1.472341 & 1.926932 \\
0.005 & 73.6061 & 1 & 1.461107 & 1.897653 \\
0.006 & 68.1137 & 1 & 1.452253 & 1.874747 \\
0.007 & 63.8318 & 1 & 1.444972 & 1.856018 \\
0.008 & 60.3648 & 1 & 1.438802 & 1.840231 \\
\hline
\end{tabular}

Calculation of the overall efficiency of the equivalent circular wing. (Table 14)

$$
\eta g=\eta f\left[\frac{\psi_{0}}{C_{0}}+\frac{\psi_{1}}{C_{1}}+\frac{\psi_{2}}{C_{2}}\right]
$$

Calculation the overall surface heat Exchange where, $X=N p \cdot l$. (Table 15)

$$
S e=(L a \cdot X \cdot H)\left(\left(\frac{\pi \cdot d e}{h \cdot l}\right)+\frac{2}{p}-\frac{\pi \cdot d e^{2}}{2 \cdot p \cdot h \cdot l}\right)
$$

Calculating the overall coefficient of heat transfer function and pitch $\mathrm{Np}$

$$
U=\frac{1}{\frac{S e}{S i}\left(\frac{1}{h i}+\frac{e t}{k t}\right)+\frac{1}{h e}\left[1-\frac{S s}{S e}(1-\eta g)\right]}
$$

where,

$$
\begin{gathered}
S i=\pi \cdot \operatorname{dik} \cdot L a \cdot N a \cdot N p \\
S S=2 \cdot \frac{L a}{p}\left(X \cdot H-N p \cdot N a \cdot\left[\frac{\pi \cdot d e^{2}}{4}\right]\right)
\end{gathered}
$$

$* k t=$ conductivity of tube, $e_{t}=$ Thickness of the wall of tube.
**The value $\frac{e t}{k t}$ is neglected as being small compared to $\frac{1}{h i}$. (Table 16)

Calculation Heat flow based on $\mathrm{Np}$ and pitch $(p)$

$$
Q=U \cdot A \cdot(\Delta T) \text {. }
$$

Table 14. Overall efficiency $(\eta g)$ as a function of $\mathrm{Np}$ and pitch $(p)$.

\begin{tabular}{cccccccc}
\hline \multicolumn{6}{c}{ Overall efficiency $(\eta g)$ as a function of Np and pitch $(p)}$. \\
\hline$\eta f$ & $(N p)=1$ & $(N p)=2$ & $(N p)=3$ & $(N p)=4$ & $(\mathrm{~Np})=5$ & $(N p)=6$ \\
0.9 & 0.412708 & 0.585084 & 0.683014 & 0.731979 & 0.761358 & 0.780944 \\
0.92 & 0.435243 & 0.607987 & 0.705014 & 0.753528 & 0.782636 & 0.802041 \\
0.93 & 0.449131 & 0.621303 & 0.717299 & 0.765297 & 0.794095 & 0.813294 \\
0.95 & 0.465868 & 0.639809 & 0.736269 & 0.784499 & 0.813437 & 0.832729 \\
0.96 & 0.476524 & 0.650701 & 0.746883 & 0.794974 & 0.823829 & 0.843066 \\
0.97 & 0.486346 & 0.660974 & 0.757071 & 0.80512 & 0.833949 & 0.853168 \\
0.98 & 0.495576 & 0.670808 & 0.766957 & 0.815031 & 0.843876 & 0.863105
\end{tabular}

Table 15. Total surface heat exchange.

\begin{tabular}{lllllll}
\hline \multicolumn{7}{c}{ TOTAL SURFACE HEAT EXCHANGE } \\
\hline$P(m)$ & $(N p)=1$ & $(N p)=2$ & $(N p)=3$ & $(N p)=4$ & $(N p)=5$ & $(N p)=6$ \\
0,002 & 60,85782 & 121,7156 & 182,5734 & 243,4313 & 304,2891 & 304,2891 \\
0,003 & 41,10674 & 82,21349 & 123,3202 & 164,427 & 205,5337 & 205,5337 \\
0,004 & 31,23121 & 62,46242 & 93,69363 & 124,9248 & 156,156 & 156,156 \\
0,005 & 25,30589 & 50,61178 & 75,91766 & 101,2236 & 126,5294 & 126,5294 \\
0,006 & 21,35567 & 42,71135 & 64,06702 & 85,42269 & 106,7784 & 106,7784 \\
0,007 & 18,53409 & 37,06818 & 55,60228 & 74,13637 & 92,67046 & 92,67046 \\
0,008 & 16,41791 & 32,83581 & 49,25372 & 65,67162 & 82,08953 & 82,08953 \\
\hline
\end{tabular}

Table 16. Overall rate of heat transfer $\left(\mathrm{W} / \mathrm{m}^{2} \mathrm{C}\right)$.

OVERALL RATE OF HEAT TRANSFER $\left(\mathrm{W} / \mathrm{m}^{2} \mathrm{C}\right)$

$P(m) \quad(N p)=1 \quad(N p)=2 \quad(N p)=3 \quad(N p)=4 \quad(N p)=5 \quad(N p)=6$

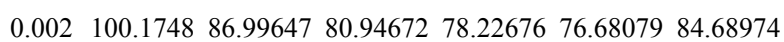

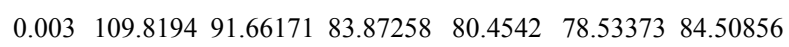

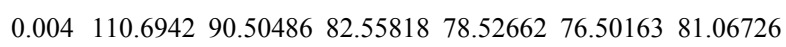

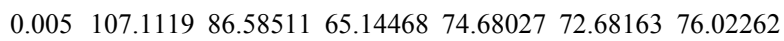

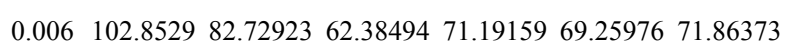

$\begin{array}{lllllll}0.007 & 98.2215 & 78.86459 & 72.69445 & 67.83032 & 65.9839 & 68.03117\end{array}$

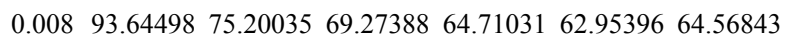


where

$A=S e=$ Overall surface heat exchange

$\Delta T=T m l=$ Difference logarithmic mean temperature.

(Table 17)

Calculation $U \cdot N p \cdot S t_{1}$ for comparison of the relationship $\left(\mathrm{Q} /{ }^{\circ} \mathrm{C}\right)$ as a function of $N p$ and the pitch between wings.

$$
\begin{aligned}
& Q=U \cdot N p \cdot S t_{1} \cdot\left(\Delta \theta_{m l}\right) \\
& \therefore U \cdot N p \cdot S t_{1}=\frac{Q}{\Delta \theta_{m l}}=\frac{250998,75}{50.85}=4935,724 \mathrm{~W} /{ }^{\circ} \mathrm{C}
\end{aligned}
$$

Where:

$S t=$ Overall surface heat exchange with respect to pitch

$N p=$ Pitch number of tubes in the direction of seconddary flow. (Table 18)

Selection based on the results obtained in the above table $U \cdot N p \cdot S t_{1}=\frac{Q}{\Delta \theta_{m l}}$ can be compared to select the exchange with their respective sizes, data are compared $4935,724059 \approx 5300,392$

This variation is important for any factor that our team

Table 17. Values of heat flow $(Q)$.

\begin{tabular}{lllllllll}
\hline \multicolumn{7}{c}{ Heat flow $(Q)$} \\
\hline$P(m)$ & $(N p)=1$ & $(N p)=2$ & $(N p)=3$ & $(N p)=4$ & $(N p)=5$ & $(N p)=6$ \\
0.002 & 310024.2 & 538478.9 & 751549.4 & 968394.7 & 1186571 & 1310502 \\
0.003 & 229568.8 & 383223.1 & 525987 & 672732.6 & 820842.8 & 883292.4 \\
0.004 & 175806.2 & 287482.5 & 393360.6 & 498868.8 & 607505.5 & 643761.5 \\
0.005 & 137841.4 & 222851.5 & 251502.6 & 384421.9 & 467667.2 & 489164.7 \\
0.006 & 111699.4 & 179689.6 & 203252.1 & 309259.2 & 376084.1 & 390223.8 \\
0.007 & 92576.03 & 148663.4 & 205548.6 & 255726.6 & 310956.7 & 320604.8 \\
0.008 & 78184.91 & 125570.7 & 173511.9 & 216108.5 & 262803.7 & 269543.4 \\
\hline
\end{tabular}

Table 18. Values of $U \cdot N p \cdot S t_{1}\left(\mathrm{~W} /{ }^{\circ} \mathrm{C}\right)$.

\begin{tabular}{llllllll}
\hline \multicolumn{6}{c}{$U \cdot N p \cdot S t_{1}$} & $\left(\mathrm{~W} /{ }^{\circ} \mathrm{C}\right)$ \\
\hline$P(m)$ & $(N p)=1$ & $(N p)=2$ & $(N p)=3$ & $(N p)=4$ & $(N p)=5$ & $(N p)=6$ \\
0,002 & 6096,42 & 10588,83 & 14778,72 & 19042,84 & 23333,13 & 25770,16 \\
0,003 & 4514,317 & 7535,829 & 10343,19 & 13228,84 & 16141,33 & 17369,36 \\
0,004 & 3457,113 & 5653,152 & 7735,176 & 9809,925 & 11946,19 & 12659,14 \\
0,005 & 2710,561 & 4382,226 & 4945,632 & 7559,402 & 9196,366 & 9619,099 \\
0,006 & 2196,494 & 3533,477 & 3996,817 & 6081,377 & 7395,444 & 7673,492 \\
0,007 & 1820,446 & 2923,367 & 4041,977 & 5028,693 & 6114,758 & 6304,48 \\
0,008 & 1537,454 & 2469,264 & 3411,996 & 4249,631 & 5167,861 & 5300,392 \\
\hline
\end{tabular}

needs to transfer more heat.

The selection of compact heat exchanger required to have 6 columns of tubes $(N p=6)$ in the direction of flow and pitch inlet wings of $8 \mathrm{~mm}$, so $X$ will be worth

$$
X=1 x N p=(0.008)(6)=0.18 \mathrm{~m}
$$

The dimensions of the compact heat exchanger will $(L)$ $x(H) x(X),(1.40 \times 1.15 \times 0.18) \mathrm{m}$.

With piping HWG $22 d e=12.7 \mathrm{~mm}$ y $d i=11.28 \mathrm{~mm}$ The thick aluminum wings $e=0.3 \mathrm{~mm}$ and $K=203.52$ $\mathrm{W} / \mathrm{m}$ C. In Table 19 are shown characteristics of the exchanger.

\section{Conclusions}

This paper identifies the advantages of having the appropriate exchanger with working conditions, environmental conditions and economic aspects, it is also necessary to mention the following regarding the general utility of this work.

- In addition to the thermal design, mechanical design of heat exchangers is also a part of it. The mechanical design is done under the ASME Section VIII, which is entitled "Pressure Vessel Design"

- Although the subject of this work is the design of heat exchangers, which, as noted was achieved successfully, the utility of it is wider and there are several methods

\begin{tabular}{|c|c|c|}
\hline \multicolumn{3}{|c|}{ CHARACTERISTICS OF THE EXCHANGE } \\
\hline de: & Outside diameter of the tubes & $12.7 \mathrm{~mm}$ \\
\hline$d i:$ & Internal diameter of the tubes. & $11.28 \mathrm{~mm}$ \\
\hline La: & Wings tube length & $1.40 \mathrm{~m}$ \\
\hline$L:$ & Overall length of the tube. & $1.40 \mathrm{~m}$ \\
\hline$N_{a}:$ & Number of vertical tubes in the exchanger. & 29 tubos \\
\hline$N_{p}:$ & $\begin{array}{l}\text { Number of tubes in the direction of secondary } \\
\text { flow. }\end{array}$ & 6 tubos \\
\hline$N:$ & Overall number of tubes ( $N=N a \cdot N p$ ). & 174 tubos \\
\hline$h$ : & $\begin{array}{l}\text { Wheelbase of the tubes in the vertical direc- } \\
\text { tion. }\end{array}$ & $0.04 \mathrm{~m}$ \\
\hline$l:$ & $\begin{array}{l}\text { Wheelbase of the tubes in the direction of } \\
\text { secondary flow. }\end{array}$ & $0.08 \mathrm{~m}$ \\
\hline$n:$ & Number of wings $(n=L a / p)$. & 175 wings \\
\hline$p:$ & Wings pitch (from center to center of wings) & $0.008 \mathrm{~m}$ \\
\hline$e:$ & Wing thickness. & $0.3 \mathrm{~mm}$ \\
\hline$H:$ & Height of heat exchanger (continuous wing) & $1.15 \mathrm{~m}$ \\
\hline$X:$ & $\begin{array}{l}\text { Heat exchanger width (within the meaning } \\
\text { of secondary flow). }\end{array}$ & $0.18 \mathrm{~m}$ \\
\hline
\end{tabular}
for the design of heat exchangers.

Table 19. Characteristics of the exchange. 
- While this paper addresses just one example of a heat exchanger, this could vary, the same results in the thermal design, these variations are affected primarily for economic reasons or space.

\section{References}

[1] D. Q. Kern, "Procesos de Transferencia de Calor," 31th Reimpresión, CECSA, México, 1999.

[2] F. P. Incropera and D. P. Dewitt, "Fundamentals of Heat and Mass Transfer," 5 th Edition, Wiley, New York, 2002.

[3] M. Serna and A. Jimenez, "A Compact Formulation of Bell-Delaware Method for Heat Exchanger Desing and Optimation," Chemical Engineering Research and Desing,
Vol. 83, (A5), 2005, pp. 539-550.

[4] R. K. Shah, P. Dusan and D. P. Sekulic, "Fundamentals of Heat Exchanger Design," John Wiley \& Sons, 2003.

[5] Z. H. Ayub, "A New Chart Method for Evaluating Single-Phase Shell Side Heat Transfer Coefficient in a Single Segmental Shell and Tube Heat Exchanger," Applied Thermal Engineering, Vol. 25, 2005, pp. 2412-2420.

[6] Y. A. Kara and Ö. Güraras, "A Computer Program for shell- and-tube Heat Exchanger," Applied Thermal Engineering, Vol. 24, 2004, pp 1797-1805.

[7] R. Castillo, "Diseño Computacional de Intercambiadores de Calor de Coraza y Tubos por el Método Delaware," Tesis de Maestría, Instituto Politécnico Nacional, México, 1999. 